\title{
Preface with a Biography of Professor Jiaqi Mo
}

\author{
Xiu Chen ${ }^{1}$ - Songlin Chen ${ }^{2} \cdot$ Xiang Zhang $^{3}$
}

Published online: 29 December 2017

(C) Springer International Publishing AG, part of Springer Nature 2017

During June 24th to 28th, 2016, Chinese Society of Singular Perturbation, of Chinese Mathematical Society, organized "The 2016 International Conference on Singular Perturbation Theory and its Applications (ICSPTA)", which was taken place at Hefei University, Hefei, Anhui Province, P.R. China (Fig. 1). This conference is the second of a series. The first one took place in Shanghai in 2010. The main goal of the conference is to bring together experts to share ideas and results on recent developments in the theory of singular perturbation and its applications. The conference mainly focused on the geometric theory of singular perturbations, asymptotic methods, multiple scales, dynamics with singular perturbations and their applications in physics, fluids, mechanics, biomathematics and so on.

Another main aim of the conference is to celebrate the 80th birthday of Professor Jiaqi Mo from Anhui Normal University, who was advisor of us for master degree. Prof. Mo was one of the founders of the theory of singular perturbation in China, he has made many fundamental contributions to the development of this theory and its applications in China. His works are mainly related to singularly perturbed ordinary

Xiang Zhang

xzhang@sjtu.edu.cn

Xiu Chen

chenxiu@hfuu.edu.cn

Songlin Chen

slchen@ahut.edu.cn

1 Department of Mathematics and Physics, Hefei University, Hefei 230601, Anhui, People's Republic of China

2 School of Mathematics and Physics, Anhui University of Technology, Maanshan 243002, Anhui, People's Republic of China

3 School of Mathematical Sciences, MOE-LSC, Shanghai Jiao Tong University, Shanghai 200240, People's Republic of China 


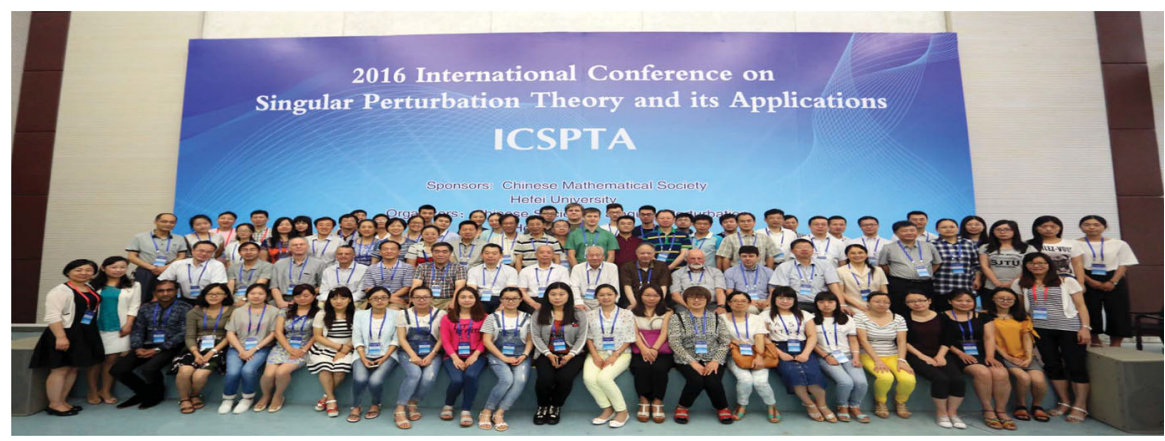

Fig. 1 ICSPTA at Hefei, China

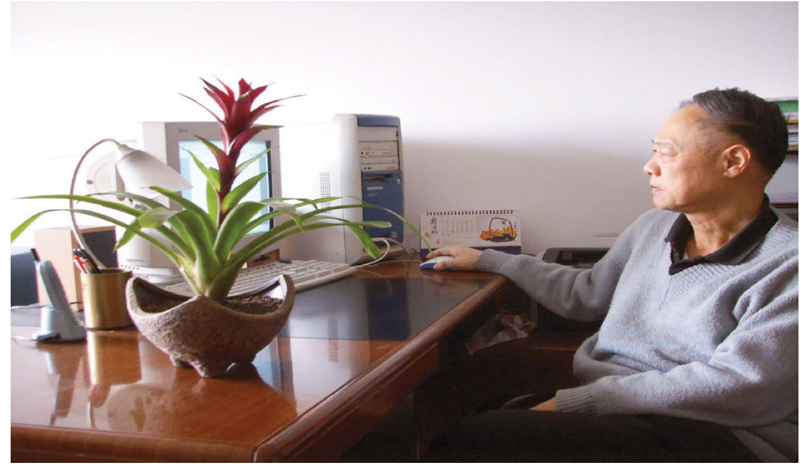

Fig. 2 Prof. Jiaqi Mo in working

and partial differential equations, and their applications in physics, mechanics and astronomy and so on.

This issue in Qualitative Theory of Dynamical Systems collects the papers for this conference on singular perturbation theory, its applications and other relative fields, which celebrate the 80th birthday of Professor Jiaqi Mo (Fig. 2).

Jiaqi Mo was born in March, 1937, in Zhenjiang, Jiangsu Province, China. He was enrolled as a college student in Department of Mathematics, Fudan University in 1955. From then he became famous in Fudan university not only his genius performance in study but also as a nice player on foot ball (the leader of football team in Fudan university). When he was a senior college student, because of his excellent performance he was allocated in the group of partial differential equations for studying partial differential equations with Professor Furu Jiang. From then, his study and research has being concentrated on partial differential equations together with ordinary differential equations involving singular perturbation.

In 1960 he joined Anhui Normal University. Starting from 1986 Prof. Mo had Xiu Chen as his first master student, and then Songli Chen and Zhiqi Huang in the second year, Qilin Liu and Xiang Zhang in the third year, and so on. Jiaqi was promoted as a full professor in mathematics of Anhui Normal University in 1987. He was also hired as adjunct professors by Shanghai University and Shanghai Jiao Tong University 
respectively, and taught courses there for masters and $\mathrm{Ph} . \mathrm{D}$. students, together with Professor Furu Jiang as coadvisors of some graduate students (e.g. Hanlin Zhang) in Shanghai University, and also did collaborate research with professors there on the theory of singular perturbation. In fact, Shanghai University and Shanghai Jiao Tong University both offered him professor positions in their universities, but Jiaqi euphemistically declined those offers in order for concentrating on development of mathematics in Anhui Normal University.

Prof. Mo was awarded as a State Council special allowance experts. He was also awarded by the Ministry of Education of China and Anhui provincial government in nine times for Scientific and Technological Achievements, Scientific and Technological Progress, and Natural Science. He has been granted nine times by the National Natural Science Foundation of China including two major projects. Jiaqi has published more than 500 papers, in which more than 150 papers were included in SCI and EI, and published also some books in Chinese and so on. He was ranked in the annual number one among the Chinese specialists several times for the number of publications published in the national core journals in those years released by Chinese Academy of Sciences. In 2012 one of his papers was nominated as 'the most influential top 100 domestic academic papers of China' and 'Top Runner 5000-top quality academic papers in Chinese academic journals' by the Ministry of Science and Technology of China. Prof. Mo is now a member of the Consultant Committee of Chinese Society of Singular Perturbation.

As one of founders of the theory of singular perturbation in China, since 1980 Prof. Mo has always being dedicated to the study of the theory of singularly perturbed differential equations and their applications, and obtained lots of interesting and important results.

Prof. Mo has being systematically investigated asymptotic expressions and their uniformly validity of solutions having boundary and inner layer phenomena for singularly perturbed ordinary differential equations [1-9], partial differential equations [10-12] and integral equations [13] with multiple parameters via the theory of differential inequalities and functional analysis [14,15]. For initial and boundary value problems of singularly perturbed reaction diffusion equations [13,16-29], and evolution equations [30,31], by constructing local coordinate systems he obtained asymptotic expressions and error estimation of generalized solutions. By establishing moving coordinate frame, he got valid asymptotic solutions of a class of boundary value problem of elliptic equations [32-37]. Prof. Mo has successfully applied the theory and methods in singular perturbation to study mathematical models from biomathematics [17,38,39], El Niño vibrator [40-44], atmospheric science [45-49], soliton wave [50-55] and so on, and achieved many new, interesting and important results, for example, the valid asymptotic solutions of the perturbed forced Klein-Gordont equations [56], instead of the classical methods, for instance, inverse scattering method and deformed maps, used in the investigation of those equations. His research has now lots of followers.

For pushing development of singular perturbation theory in China, he has organized many seminars, lectures and conferences, which accelerate interchange of ideas and knowledge and cooperation among scholars in the theory of singular perturbation (Fig. 3). In order to help Chinese scholars collecting references in the field of singular 


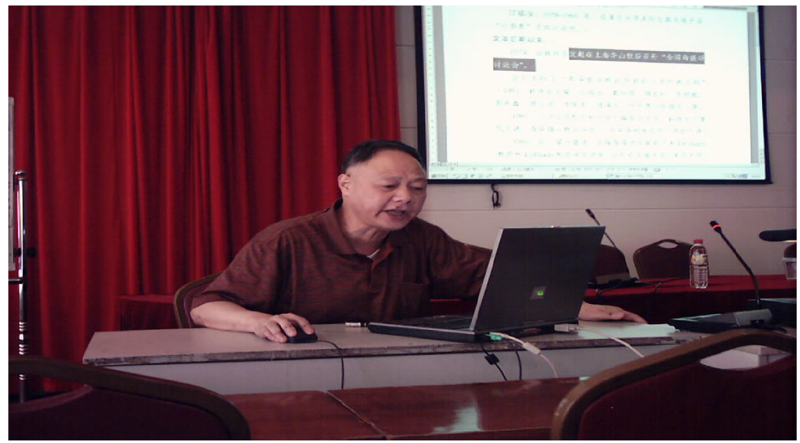

Fig. 3 Prof. Jiaqi Mo in presenting a talk

perturbation, he and his collaborators founded 'An electronic systems on abstracts of papers in singular perturbations and related problems'.

For praising Mo's important contributions to the development and propagation of the theory of singular perturbation in China, during the "2012 National Conference on Singular Perturbation Theory and its Application, and celebrating the 100th Anniversary of the Birth of Qian Weichang, the most early Founder of Chinese Singular Perturbation", Chinese Society of Singular Perturbation awarded Professor Jiaqi Mo as "One of the outstanding Contributors to Chinese Singular Perturbation".

Not only in research, but also in teaching, Jiaqi had many good ideas to encourage and stimulate young students and scholars for studying hard and concentrating on their research. He did his best to help youth such that they will become outstanding scientists. Because of his enthusiasm and furtherance to young in research he has being received highly respect and esteem by colleagues in China.

\section{References}

1. Han, X., Shi, L., Mo, J.: Generalized solution of nonlinear nonlocal singularly perturbed problems with two parameters. Adv. Math. (Beijing) 45(1), 95-101 (2016). (in Chinese)

2. Chen, L., Mo, J.: Positive solution of singularly perturbed Dirichlet problem with singularities. Acta Math. Appl. Sin. Engl. Ser. 30(3), 611-616 (2014)

3. Mo, J.: Singularly perturbed solution of boundary value problem for nonlinear equations of fourth order with parameters. Adv. Math. (Beijing) 39(6), 736-740 (2010)

4. Mo, J.: Asymptotic property of solutions for a class of nonlinear singularly perturbed boundary value problem with two parameters. Adv. Math. (Beijing) 39(2), 217-223 (2010). (in Chinese)

5. Mo, J.: Asymptotic property of a semi-linear singularly perturbed problem with two parameters. Acta Math. Appl. Sin. 32(5), 903-911 (2009). (in Chinese)

6. Mo, J.: A class of shock solution for quasilinear Robin problems. Acta Math. Sci. 28A(5), 818-822 (2008). (in Chinese)

7. Mo, J., Zhu, J., Wang, H.: Asymptotic behavior of the shock solution for a class of nonlinear equations. Progress Nat. Sci. 13(10), 768-770 (2003)

8. Mo, J.: The singularly perturbed nonlinear boundary value problems. Appl. Math. J. Chin. Univ. 15(4), 377-382 (2000)

9. Mo, J.: A singularly perturbed nonlinear boundary value problem. J. Math. Anal. Appl. 178(1), 289-293 (1993)

10. Mo, J.: Analytic solution for a class of generalized Sine-Gordon perturbation equation. Acta Phys. Sin. 58(5), 2930-2933 (2009). (in Chinese) 
11. Mo, J.: Variational iteration solving method for a class of generalized Boussinesq equation. Chin. Phys. Lett. 26(6), 060202 (2009)

12. Mo, J., Chen, Y.: Approximate solution of homotopic mapping for generalized Boussinesq equation. Acta Phys. Sin. 58(7), 4379-4382 (2009). (in Chinese)

13. Mo, J.: A class of singularly perturbed reaction differential integral differential system. Acta Math. Appl. Sin. 15(1), 18-23 (1999)

14. Mo, J.: Homotope method of solutions on gain fluence of a laser pulse amplifier. Sci. China Ser. G 39(5), 658-661 (2009)

15. Mo, J.: Homotopic mapping solving method for gain fluency of a laser pulse amplifier. Sci. China Ser. G 52(7), 1007-1010 (2009)

16. Yao, J., Mo, J.: The interior and boundary layers solution for reaction diffusion equations. Adv. Math. (Beijing) 42(2), 159-164 (2013)

17. Wen, Z., Mo, J.: Singular perturbation for reaction diffusion equations of activator inhibitor systems. Adv. Math. (Beijing) 41(4), 455-462 (2012)

18. Mo, J.: A class of singularly perturbed differential-difference reaction diffusion equation. Adv. Math. (Beijing) 38(2), 227-231 (2009)

19. Mo, J.: Nonlinear singularly perturbed reaction diffusion problems with ulter parabolic climiting equations. Adv. Math. (Beijing) 37(1), 83-91 (2008)

20. Mo, J., Chen, X.: The nonlinear singularly perturbed nonlocal reaction diffusion systems. Acta Math. Appl. Sin. 24(4), 553-562 (2008)

21. Mo, J.: Singurlar perturbation of weaken nonlinear reaction diffusion equations with boundary perturbation. Appl. Math. Mech. 29(8), 1003-1008 (2008). (in Chinese)

22. Mo, J., Zhang, W., Chen, X.: Asymptotic behavior for a class of nonlinear reaction diffusion system with jump layer. Adv. Math. (Beijing) 36(5), 631-636 (2007)

23. Mo, J., Zhang, W., He, M.: Asymptotic method of traveling wave solutions for a class of nonlinear reaction diffusion equation. Acta Math. Sin. 27B(4), 777-780 (2007)

24. Mo, J., Han, X., Chen, S.: The singularly perturbed nonlocal reaction diffusion system. Acta Math. Sci. 22B(4), 549-556 (2002)

25. Mo, J.: The singularly perturbed problem for combustion reaction diffusion. Acta Math. Appl. Sin. 17(2), 255-259 (2001)

26. Mo, J.: A class of singularly perturbed problems with nonlocal reaction diffusion equation. Adv. Math. (Beijing) 27(1), 53-58 (1998)

27. Mo, J., Xu, Y.: A class of singularly perturbed nonlinear reaction diffusion integral-differential system. Acta Math. Appl. Sin. 17(2), 278-286 (1994). (in Chinese)

28. Mo, J.: Singular perturbation of initial-boundary value problems for a class of reaction diffusion systemsm. Appl. Math. Mech. 12(4), 399-408 (1991)

29. Mo, J.: Singular perturbation for a class of nonlinear reaction diffusion systems. Sci. China Ser. A 32(11), 1306-1315 (1989)

30. Mo, J.: Generalized iterative solutions of a class of nonlinear perturbed evolution equations. Acta Phys. Sin. 60(2), 020202 (2011). (in Chinese)

31. Lin, S., Mo, J.: Asymptotic solutions of a class of nonlinear hyperparabolic equations. Adv. Math. (Beijing) 39(4), 472-476 (2010). (in Chinese)

32. Han, X., Wang, W., Mo, J.: Solution of singularly perturbed boundary value problem for nonlinear higher order elliptic partial differential equations with two parameters. Adv. Math. (Beijing) 44(6), 931-938 (2015)

33. Mo, J.: Asymptotic solutions of singularly perturbed semi-linear elliptic equations with double parameters. Chin. Ann. Math. 31A(3), 331-336 (2010). (in Chinese)

34. Mo, J., Zhang, W., Chen, X.: Solvability for nonlinear elliptic equation with boundary perturbation. Appl. Math. J. Chin. Univ. 22B(4), 421-424 (2007)

35. Mo, J., Shao, S.: The singularly perturbed boundary value problems for higher-order semilinear elliptic equations. Adv. Math. (Beijing) 30(2), 141-148 (2001)

36. Mo, J.: The nonlocal boundary value problems of nonlinear elliptic systems in unbounded domains. Appl. Math. Comput. 86(2/3), 115-121 (1997)

37. Mo, J., Cheng, Y.: The singular perturbation for a class of similinear elliptic equations. Acta Math. Sci. 12, 52-54 (1992). (in Chinese)

38. Mo, J., Yao, J., Wang, H.: The nonlinear species group singularle perturbed Robin problems for reaction diffusion system. J. Biomath. (Anshan) 22(2), 193-199 (2007) 
39. Mo, J., Wang, H.: Nonlinear singular perturbed approximate solution for generalized Lotke-Volterra ecological model. Acta Ecol. Sin. 27(10), 4366-4370 (2007)

40. Mo, J., Lin, W., Wang, H.: A class of homotopic solving method for ENSO model. Acta Math. Sin. 29(1), 101-110 (2009)

41. Liu, S., Lin, Y., Wang, H., Mo, J.: Perturbed solution of sea-air oscillator for the El Niño/La NinaSouthern oscillation mechanism. Acta Oceanol. Sin. 28(3), 1-4 (2009)

42. Mo, J., Lin, W.: The homotopic method of travelling wave solution for El Niño tropic sea-air coupled oscillators. Chin. Phys. 17(3), 743-746 (2008)

43. Mo, J., Lin, W., Wang, H.: A perturbed solution of sea-air oscillator for the ENSO mechanism. J. Syst. Sci. Complex. 18(2), 219-223 (2005)

44. Mo, J., Lin, W., Zhu, J.: The perturbed solution of sea-air oscillator for ENSO model. Progress Nat. Sci. 14(6), 550-552 (2004)

45. Mo, J., Lin, W.: Singularly perturbed solution in atmosphere-ocean for global climate. Chin. Geogr. Sci. 18(2), 193-196 (2008)

46. Mo, J., Lin, W., Wang, H.: Variational iteration method for solving perturbed mechanism of western boundary undercurrents in the Pacific. Chin. Phys. 16(4), 951-955 (2007)

47. Mo, J., Lin, W., Wang, H.: Variational iteration solution of a sea-air oscillator model for the ENSO. Progress Nat. Sci. 17(2), 230-232 (2007)

48. Mo, J., Lin, W.: Homotopic mapping method of solution for the sea-air oscillator model of decadal variations in subtropical cells and equatorial Pacific. Acta Phys. Sin. 56(10), 5565-5568 (2007)

49. Mo, J., Wang, H., Lin, W.: Homptopic mapping solving method for perturbed mechanism of western boundary undercurrents in equator Pacific. Chin. Geogr. Sci. 16(4), 347-350 (2006)

50. Ouyang, C., Shi, L., Wang, W., Mo, J.: The asymptotic solving method of solitary wave for the nonlinear forced disturbed Klein-Gordon equation. Chin. Ann. Math. Ser. A 38(1), 43-52 (2017). (in Chinese)

51. Mo, J.: Solution of travelling wave for nonlinear disturbed long-wave system. Commun. Theor. Phys. 55(3), 387-390 (2011)

52. Mo, J.: Soliton solution to generalized nonlinear disturbed Klein-Gordon equation. Appl. Math. Mech. 31(12), 1577-1584 (2010)

53. Mo, J., Yao, J.: Approximate solution of 2-soliton for generalized disturbed mKdV coupled system. Acta Phys. Sin. 59(8), 5190-5193 (2010). (in Chinese)

54. Mo, J.: Approximation of the soliton solution for generalized Vakhnenko equation. Chin. Phys. 18(11), 4608-4612 (2009)

55. Mo, J., Zhang, W., He, M.: The variational iteration method for the soliton solution of nonlinear generalized Landau-Ginzburg-Higgs equation. Acta Phys. Sin. 56(4), 1847-1850 (2007)

56. Mo, J., Lin, Y., Lin, W.: Homotopic mapping solving method of the reduces equation for Kelvin waves. Chin. Phys. 19(3), 030202-1-4 (2010) 